\title{
Enhancing spin-transfer torque through the proximity of quantum well states
}

\author{
Ioannis Theodonis, ${ }^{1,2, *}$ Alan Kalitsov, ${ }^{1}$ and Nicholas Kioussis ${ }^{1}$ \\ ${ }^{1}$ Department of Physics, California State University, Northridge, California 91330-8268, USA \\ ${ }^{2}$ Department of Physics, National Technical University, Zografou, Athens GR-15773, Greece
}

(Received 4 October 2007; published 11 December 2007)

\begin{abstract}
We predict that the spin-transfer, $T_{i, \|}$, and fieldlike, $T_{i, \perp}$, components of the local spin torque are dramatically enhanced in double-barrier magnetic tunnel junctions. The spin-mixing enhancement is due to the energetic proximity of majority and minority quantum well states (QWSs) of different quantum numbers within the bias window. The local-spin-torque enhancement is not associated with a corresponding enhancement of the spinpolarized currents. $T_{i, \|}$ exhibits a switch-on and switch-off steplike bias behavior when spin-polarized QWSs enter the bias window or exit the energy band, while $T_{i, \perp}$ changes sign between switch-on biases. The net $T_{\perp}$ exhibits an anomalous angular behavior due to the bias interplay of the bilinear and biquadratic effective exchange couplings.
\end{abstract}

DOI: 10.1103/PhysRevB.76.224406

PACS number(s): 85.75.- d, 72.10.-d, 72.25.-b, 73.40.Gk

\section{INTRODUCTION}

Spintronics involve the exploitation of the quantummechanical spin degree of freedom to provide new functionalities beyond conventional electronics. ${ }^{1}$ One effect that has its roots on the electron's spin is the torque exerted on the magnetization of a nanometer-scale free ferromagnet (FM) by a spin-polarized current, originating from a preceding noncollinear pinned FM. ${ }^{2-4}$ This torque can be decomposed into a fieldlike and a spin-transfer component, ${ }^{5}$ both orthogonal to the magnetic moment of the free FM but with different influences on its dynamics. ${ }^{6}$ Recent ferromagnetic resonance experiments provide a useful tool to study the role of each component. ${ }^{7,8}$ At sufficiently high current densities, the spintransfer torque leads to current-induced magnetization switching (CIMS). ${ }^{9-11}$ Reduction of the high critical current for CIMS is necessary for spin-transfer controlled magnetic memories. $^{12}$

Double-barrier magnetic tunnel junctions (DBMTJs) consist of a central metallic layer between two insulating barriers and two FM electrodes. The tunneling magnetoresistance (TMR) can be dramatically enhanced in collinear DBMTJs by the presence of quantum well states (QWSs) under appropriate resonant conditions. ${ }^{13-16}$ Recently, the discrete energy spectrum of FM nanoparticles has been shown to enhance spin accumulation and to control the bias dependence of the TMR. ${ }^{17}$ While the physics in collinear DBMTJs has been studied extensively, ${ }^{13-16}$ the effect of spin-polarized QWSs (SPQWSs) on the spin torque in noncollinear DBMTJs remains an unexplored area thus far.

The objective of this work is to present a study of the effect of SPQWSs on both the spin-transfer, $T_{\|}$, and fieldlike, $T_{\perp}$, components of the spin torque under external bias. The calculations are based on the tight-binding method and the nonequilibrium Keldysh formalism. We predict that both components of the local spin torque can be dramatically enhanced when majority and minority QWS energies of different quantum numbers are in close proximity and lie within the bias window. It should be emphasized that the local-spintorque enhancement is not associated with an enhancement of the corresponding spin-polarized currents. The low- temperature bias dependence of the local spin-transfer torque $T_{i, \|}$ exhibits a switch-on and switch-off steplike behavior when the SPQWSs enter the bias window or exit the energy band, respectively, similar to that of the spin-polarized currents. On the other hand, $T_{i, \perp}$ changes sign between the majority and minority switch-on values of bias. We demonstrate that the bias behavior of $T_{i, \|}$ and $T_{i, \perp}$ can be derived analytically using a single-site central FM region. The net $T_{\perp}$, pertinent to the nonequilibrium interlayer exchange coupling, $E_{\mathrm{XC}}$, exhibits an anomalous angular behavior due to the interplay of the bilinear and biquadratic effective exchange couplings which have different bias behaviors.

The structure of this paper is as follows. In Sec. II, we introduce the basic model and outline the computational approach of the nonequilibrium spin torque. In Sec. III, we present and discuss the results of the calculations. Finally, Sec. IV includes a brief statement of the conclusions.

\section{METHOD: FORMALISM}

Figure 1 shows the one-dimensional $\mathrm{FM} / \mathrm{I} / \mathrm{FM} / \mathrm{I} / \mathrm{FM}$ DBMTJ system, consisting of a central $(C)$ FM nanowire containing $N_{C}$ atomic sites (ASs) connected to the left $(L)$ and right $(R)$ FM electrodes through two thin symmetric nonmagnetic tunneling barrier nanowires $I$ of $N=2$ ASs. The

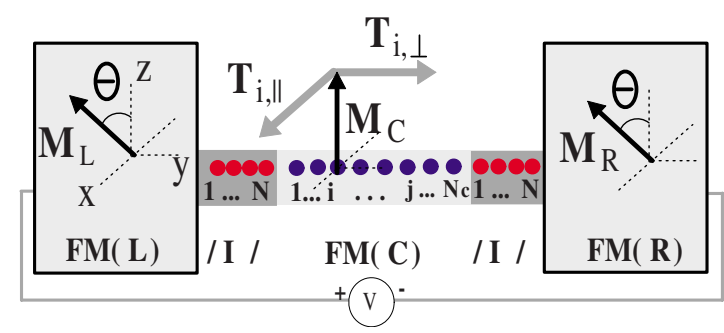

FIG. 1. (Color online) Schematic of the DBMTJ consisting of a FM central wire of $N_{C}$ atomic sites, connected to left and right FM leads through the tunneling barriers $I$ of $N$ sites. The spin-transfer (fieldlike) $T_{i, \|}\left(T_{i, \perp}\right)$ components of the torque lies in the $x(y)$ directions. 
magnetization of the central FM, $\mathbf{M}_{C}$, is along the $z$ axis of the coordinate system shown in Fig. 1. The magnetization of the FM leads, $\mathbf{M}_{L(R)}$, lies in the $x-z$ plane, i.e., it is rotated by angle $\theta$ around the wire axis $y$. The Hamiltonian of the system is

$$
H=H_{L}+H_{M}+H_{R}+H_{L, M}+H_{R, M}+\text { H.c., }
$$

where $H_{M}=H_{I}+H_{C}+H_{I, C}+$ H.c. is the Hamiltonian of the middle $(M)$ multilayer $I / \mathrm{FM} / I$ region, and $H_{L(R), M}$ and $H_{I, C}$ are the coupling Hamiltonians at the $L(R) / M$ and $I / C$ interfaces, respectively. The Hamiltonian for each FM region $H_{\alpha}$, $\alpha=L, R$, and $C$, is described by a one-dimensional singleorbital tight-binding model neglecting the in-plane $k$ dependence, which includes a nearest-neighbor (NN) spinindependent hopping term, $t_{\alpha}$, and a spin-dependent on-site energy term, $\varepsilon_{\alpha}^{\sigma}$, i.e.,

$$
H_{\alpha}=\sum_{\sigma, i} \varepsilon_{\alpha}^{\sigma} c_{i}^{\dagger} c_{i}+\sum_{i} t_{\alpha} c_{i}^{\dagger} c_{i+1}+\text { H.c. }
$$

The Hamiltonian $H_{I}$ for the barriers is identical to $H_{\alpha}$, but where one replaces the hopping term $t_{\alpha}$ with $t_{I}$ and the spindependent on-site energy $\varepsilon_{\alpha}^{\sigma}$ with the spin-independent $\varepsilon_{I}$. The coupling Hamiltonian of the $\mathrm{FM} / I$ interface is $H_{\alpha, I}$ $=t_{\alpha / I} c_{\alpha}^{\dagger} c_{I}$. The exchange splitting, $\Delta_{\alpha}=\varepsilon_{\alpha}^{\uparrow}-\varepsilon_{\alpha}^{\downarrow}$, is identical in all FMs, $\Delta_{I}=0, \varepsilon_{\alpha}^{\uparrow}-E_{F}=0.318 \mathrm{eV}, \varepsilon_{\alpha}^{\downarrow}-E_{F}=0.736 \mathrm{eV}$, and $\varepsilon_{I}-E_{F}=6.5 \mathrm{eV}$, where $E_{F}$ is the Fermi energy. The $t_{\alpha}$ $=0.4 \mathrm{eV}$ in all FMs, $t_{I}=1 \mathrm{eV}$ in both insulators, and $t_{\alpha / I}$ $=0.5 \mathrm{eV}$ in all $\mathrm{FM} / I$ interfaces, consistent with the $a b$ initio values for one-dimensional Co FM nanowires. ${ }^{18}$

We extend Datta's formalism ${ }^{19}$ to the case of noncollinear systems, where the scalar Green functions are replaced with $2 \times 2$ matrices in spin space. For this purpose, $H_{\alpha}$ can be expressed in the form $H_{\alpha}=\bar{H}_{\alpha}+\delta H_{\alpha}$, where

$$
\bar{H}_{\alpha}=\frac{1}{2}\left(\varepsilon_{\alpha}^{\uparrow}+\varepsilon_{\alpha}^{\downarrow}\right)+t_{\alpha}
$$

describes the spin-average part of $H_{\alpha}$ and

$$
\delta H_{\alpha}=\frac{1}{2}\left(\varepsilon_{\alpha}^{\uparrow}-\varepsilon_{\alpha}^{\downarrow}\right)
$$

is the spin-splitting part of $H_{\alpha}$. The one-electron Schrödinger equation for the retarded Green function $g_{p q}^{\sigma, \sigma^{\prime}}$ in each isolated semi-infinite ferromagnetic lead becomes

$$
\begin{aligned}
\sum_{p_{1}}\left[\left(E \delta_{p p_{1}}-\bar{H}_{p p_{1}}\right) I-\delta H_{p p_{1}}\left(\begin{array}{cc}
\cos \theta & \sin \theta \\
\sin \theta & -\cos \theta
\end{array}\right)\right] \\
\times\left(\begin{array}{cc}
g_{p_{1} q}^{\uparrow \uparrow} & g_{p_{1} q}^{\uparrow \downarrow} \\
g_{p_{1} q}^{\downarrow \uparrow} & g_{p_{1} q}^{\downarrow \downarrow}
\end{array}\right)=\delta_{p q} I,
\end{aligned}
$$

where $I$ is a $2 \times 2$ unit matrix. Following Datta, ${ }^{19}$ we find that the retarded Green function of the middle region $M$ is

$$
\hat{G}_{M}=\left[E \hat{I}-\hat{H}_{M}-\hat{\Sigma}_{L}-\hat{\Sigma}_{R}\right]^{-1},
$$

where $E$ is the one-electron electron energy, $\hat{H}_{M}$ and $\hat{\Sigma}_{L(R)}$ are the $\left(2 N_{M} \times 2 N_{M}\right)$ Hamiltonian and self-energy matrices, respectively, and $N_{M}=2 N+N_{C}$ is the number of atomic sites in the middle region. The only nonzero elements of $\hat{\Sigma}_{L(R)}$ are

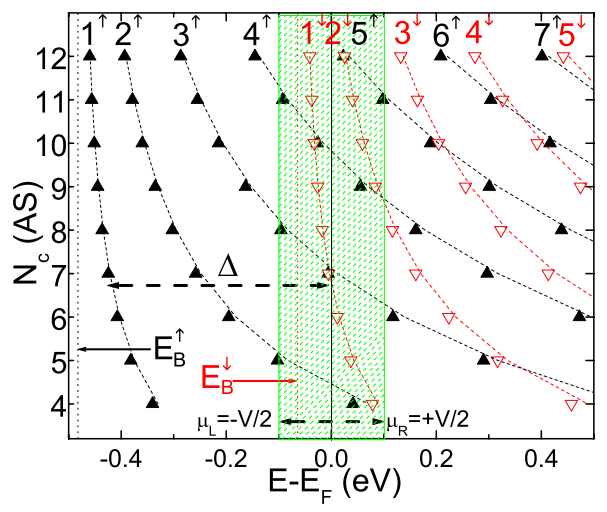

FIG. 2. (Color online) SPQWS energy positions, $E^{n^{\sigma}}$, as a function of the number of atomic sites, $N_{c}$, in the central FM region for zero bias and $\theta=0$. The bottom of the majority (minority) conduction band of the leads is denoted by $E_{B}^{\uparrow(\downarrow)}$ and $\Delta$ is the exchange splitting, denoted by the dashed horizontal arrow. At finite bias $V$, the chemical potentials of the $L, R$ leads are shifted by $e V=\mu_{R}$ $-\mu_{L}$ around the Fermi energy.

the $2 \times 2$ self-energy matrices at the interfacial sites,

$$
\widetilde{\Sigma}_{L(R)}(\theta)=t_{\alpha / I}^{2} \widetilde{g}_{L(R)}(\theta),
$$

where $\widetilde{g}_{L(R)}(\theta)$ are the retarded surface $2 \times 2$ Green's function matrices of the isolated $L(R)$ lead, determined from Eq. (5). The nonequilibrium Green's functions can be determined by solving the kinetic equation ${ }^{19}$

$$
\hat{G}_{M}^{<}=i \hat{G}_{M} \hat{\Sigma}^{<} \hat{G}_{M}^{\dagger},
$$

where $\hat{\Sigma}^{<}=f_{L}\left(\hat{\Sigma}_{L}^{\dagger}-\hat{\Sigma}_{L}\right)+f_{R}\left(\hat{\Sigma}_{R}^{\dagger}-\hat{\Sigma}_{R}\right)$ is the nonequilibrium self-energy matrix and $f_{L(R)}$ are the Fermi-Dirac distribution functions of the $L(R)$ leads.

The local spin-transfer torque $\mathbf{T}_{i}$ exerted on the local moment at site $i$ in the central FM region is ${ }^{20}$

$$
\mathbf{T}_{i} \equiv-\nabla \cdot \boldsymbol{I}^{(s)}=\boldsymbol{I}_{i-1, i}^{(s)}-\boldsymbol{I}_{i, i+1}^{(s)},
$$

where

$$
\boldsymbol{I}_{i, i \pm 1}^{(s)}=\frac{t_{C}}{4 \pi} \int \operatorname{Tr}_{\sigma}\left[\left(\widetilde{G}_{i, i \pm 1}^{<}-\tilde{G}_{i \pm 1, i}^{<}\right) \boldsymbol{\sigma}\right] d E
$$

is the spin current between NN sites, ${ }^{20}$ and $\boldsymbol{\sigma}=\left(\sigma_{x}, \sigma_{y}, \sigma_{z}\right)$ is a vector of the Pauli matrices. The fieldlike, $T_{i, \perp}$, and spintransfer, $T_{i, \|}$, components of the local spin torque, shown in Fig. 1, are along the $\hat{M}_{C} \times\left(\hat{M}_{L(R)} \times \hat{M}_{C}\right)$ and $\hat{M}_{C} \times \hat{M}_{L(R)}$ directions, respectively. Here, $\hat{M}_{C}$ and $\hat{M}_{L(R)}$ are unit vectors along the magnetization of the free $C$ and pinned $L(R)$ FM regions, respectively.

\section{RESULTS: DISCUSSION}

The majority (full triangles) and minority (open triangles) QWS energies relative to the Fermi energy $E^{n^{\sigma}}$ as a function of the thickness $N_{C}$ of the central FM wire are shown in Fig. 2 for QWSs between -0.5 and $0.5 \mathrm{eV}$. The numbers next to 


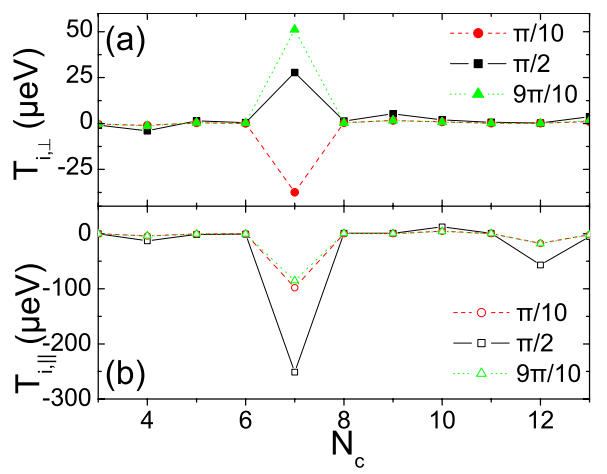

FIG. 3. (Color online) Low-temperature $(T=5 \mathrm{~K})$ and low-bias $(V=0.1 \mathrm{~V})$ (a) perpendicular, $T_{i, \perp}$, and (b) parallel, $T_{i, \|}$, components of the local spin torque vs the number of atomic sites, $N_{c}$, of the central FM region. Both components of the local spin torques are calculated on the first site in the central FM region next to the left $\mathrm{FM} / I$ interface for three values of the angle $\theta$ of $\pi / 10, \pi / 2$, and $9 \pi / 10$, respectively.

each series of data points, indicate the quantum number $n^{\sigma}$ $=1^{\sigma}, 2^{\sigma}, \ldots, N_{c}^{\sigma}$ of the SPQWSs. The dashed curves denote the SPQWS energies $E_{0}^{n^{\sigma}}=\varepsilon^{\sigma}+2 t \cos \left(n^{\sigma} \pi /\left(N_{C}+1\right)\right)$ of the isolated central FM wire. The coupling of the central region to the FM leads results in a shift and a broadening of the SPQWS energies. The bottom of the majority (minority) conduction band of the leads at zero bias is indicated by $E_{B}^{\uparrow(\downarrow)}$. Note that for $N_{c}=7$ ASs, the $n^{\uparrow}=3^{\uparrow}$ majority and $n^{\downarrow}=1^{\downarrow}$ minority QWSs are in very close proximity and they are very close to the Fermi energy. Under applied bias $V$, only the SPQWSs with energies $E^{n^{\sigma}}$ that lie within the bias window from $\mu_{L}=-\frac{e V}{2}$ to $\mu_{R}=+\frac{e V}{2}$, denoted by the shaded area in Fig. 2, contribute to the resonant tunneling.

In Figs. 3(a) and 3(b), we show the perpendicular, $T_{i, \perp}$, and parallel, $T_{i, \|}$, components of the local spin torque on the first site $(i=1)$ in the central FM region next to the left FM/I interface as a function of the thickness $N_{c}$ of the central FM region. Both local-spin-torque components are calculated at $T=5 \mathrm{~K}$ and $V=0.1 \mathrm{~V}$ for the almost parallel $\theta=\pi / 10$, perpendicular $\theta=\pi / 2$, and almost antiparallel $\theta=9 \pi / 10$ configurations. We find that $T_{i, \perp}$ and $T_{i, \|}$ are strongly enhanced for $N_{c}=7$ ASs by about 1 and 2 orders of magnitude, respectively. The local-spin-torque enhancement persists even for very small angular deviations from the parallel (circles) and antiparallel (triangles) configurations. Interestingly, $T_{i, \perp}$ for $N_{c}=7$ changes sign with increasing $\theta$, in contrast to $T_{i, \|}$.

In Figs. 4(a), 4(c), 4(b), and 4(d), we display the angular dependence of the perpendicular, $T_{i, \perp}$ (black squares) and parallel, $T_{i, \|}$ (red circles), components of the local spin torque for $N_{c}=4 \mathrm{ASs}$ and $N_{c}=7 \mathrm{ASs}$, respectively. The spin torques are evaluated on the first site in the central FM region next to the left FM/I interface at $T=5 \mathrm{~K}$ and $V=0.1 \mathrm{~V}$. In both cases [Figs. 4(b) and 4(d)], $T_{i, \|}$ exhibits a sinusoidal angular dependence similar to that in a single MTJ. ${ }^{3}$ On the other hand, when the enhancement conditions are fulfilled for $N_{c}=7 \mathrm{ASs}$ [Fig. 4(c)], $T_{i, \perp}$ exhibits a nonsinusoidal angular dependence changing sign in the $[0, \pi]$ interval.

In Figs. 5(a), 5(c), 5(b), and 5(d), we display the perpen-

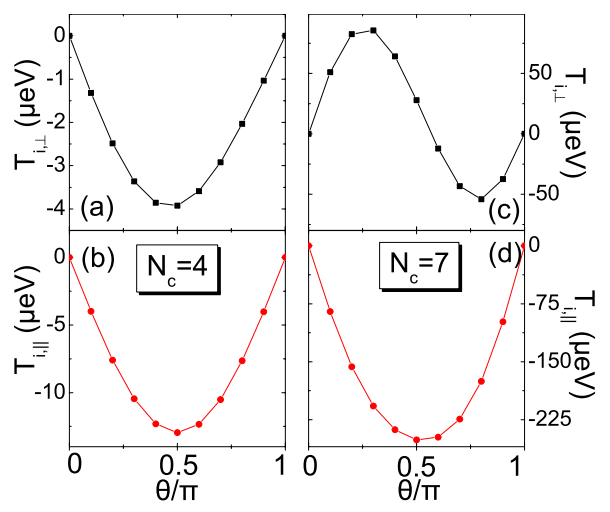

FIG. 4. (Color online) Angular dependence of $T_{i, \perp}$ (black squares) and $T_{i, \|}$ (red circles) for $N_{c}=4 \mathrm{ASs}$ in panels (a) and (b) and for $N_{c}=7 \mathrm{ASs}$ in panels (c) and (d), respectively. The local spin torque is evaluated on the first site in the central FM region next to the left $\mathrm{FM} / I$ interface at $T=5 \mathrm{~K}$ and $V=0.1 \mathrm{~V}$.

dicular, $T_{i, \perp}$ (black squares), and parallel, $T_{i, \|}$ (red circles), components of the local spin torque as a function of site $i$ in the central FM for $N_{c}=4 \mathrm{ASs}$ and $N_{c}=7 \mathrm{ASs}$, respectively. The spin torques are evaluated at $T=5 \mathrm{~K}, V=0.1 \mathrm{~V}$, and $\theta$ $=\pi / 2$. Interestingly both $T_{i, \perp}$ and $T_{i, \|}$ oscillate around zero as a function of atomic site $i$ due to the electron precession in the central FM. The number of nodes increases as the width of the FM quantum well increases. The enhancement of $T_{i, \perp}$ and $T_{i, \|}$ for $N_{c}=7$ ASs holds for all sites $i$.

In Figs. 6(a) and 6(b), we display the low-temperature $(T=5 \mathrm{~K})$ bias dependence of the spin-polarized currents $I^{\uparrow}$ and $I^{\downarrow}$ for $N_{c}=4 \mathrm{ASs}$ and $N_{c}=7 \mathrm{ASs}$, respectively, and for $\theta=\frac{\pi}{2}$. The spin-polarized currents switch on at $V_{\mathrm{on}}^{\sigma}=2 \mid E^{n_{\sigma}}$ $-E_{F} \mid$, when the $n_{\sigma}$ QWS enters the bias energy window. For $N_{c}=7 \mathrm{ASs}$, the switch on for both spin-polarized currents occur at about the same bias, $V_{\mathrm{on}}^{\uparrow} \approx V_{\mathrm{on}}^{\downarrow}$, due to the fact that $\left|E^{\downarrow}-E_{F}\right| \approx\left|E^{3^{\uparrow}}-E_{F}\right|$ in Fig. 2. Both currents decrease with increasing bias because the density of states of the minority band in the leads at $E^{n^{\sigma}}$ decreases. At the critical bias, $V_{\text {off }}^{\downarrow}$

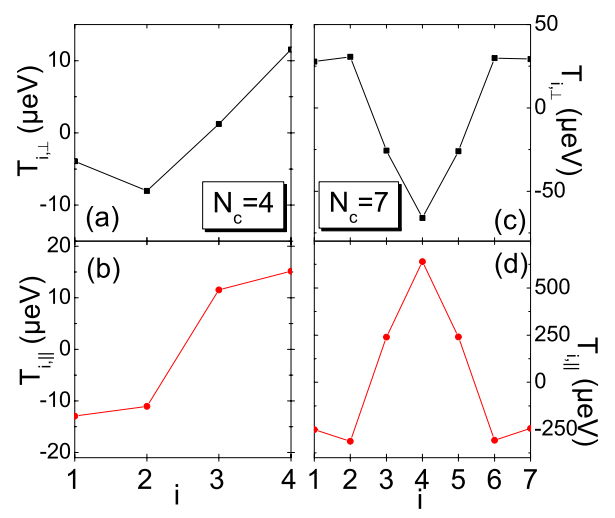

FIG. 5. (Color online) Perpendicular, $T_{i, \perp}$ (black squares), and parallel, $T_{i, \|}$ (red circles), components of spin torque as a function of site $i$ in the central FM region for $N_{c}=4$ ASs in panels (a) and (b) and for $N_{c}=7 \mathrm{ASs}$ in panels (c) and (d), respectively. The local components are calculated at $T=5 \mathrm{~K}, 0.1 \mathrm{~V}$ bias, and $\theta=\pi / 2$. 


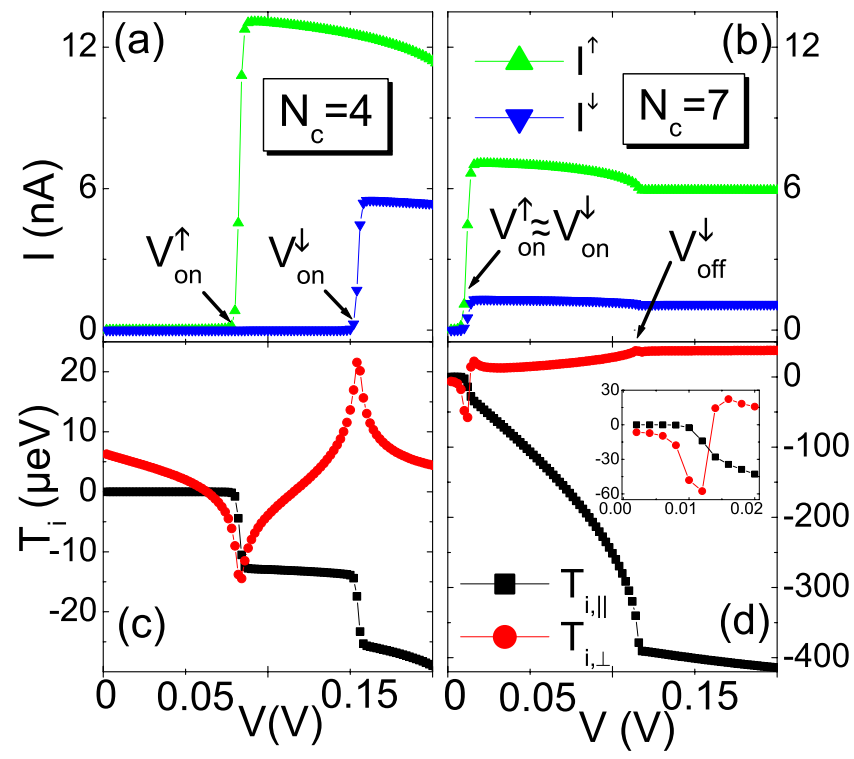

FIG. 6. (Color online) Low-temperature $(T=5 \mathrm{~K})$ bias dependence of the majority (green symbols) and minority (blue symbols) currents in the central FM for (a) $N_{c}=4 \mathrm{ASs}$ and (b) $N_{c}=7 \mathrm{ASs}$, respectively, and for $\theta=\pi / 2$. Bias dependence of $T_{i, \|}$ (black symbols) and $T_{i, \perp}$ (red symbols) on the first site in the central FM for (c) $N_{c}=4$ ASs and (d) $N_{c}=7$ ASs. The bias $V_{\text {on(off) }}^{\sigma}$ denote the switch-on (switch-off) bias.

$=2\left(E^{n^{\sigma}}+\left|E_{B}^{\downarrow}\right|\right)$, the QWS energies $E^{1^{\downarrow}}$ and $E^{3^{\uparrow}}$ are shifted below the bottom of the minority band of the lead and, hence, the minority contribution to the spin-polarized currents is switched off.

In Figs. 6(c) and 6(d), we display the low-temperature ( $T=5 \mathrm{~K}$ ) bias behavior of the parallel, $T_{i, \|}$ (black squares), and perpendicular, $T_{i, \perp}$ (red circles), components of the local spin torque on the first site in the central FM for $\theta=\frac{\pi}{2}$ and for $N_{c}=4 \mathrm{ASs}$ and $N_{c}=7 \mathrm{ASs}$, respectively. The local spintransfer component, $T_{i, \|}$, exhibits a switch on bias behavior at $V_{\text {on }}^{\uparrow}$ and at $V_{\text {on }}^{\downarrow}$, similar to that of the spin-polarized currents in Fig. 6(a). On the other hand, $T_{i, \perp}$, which is nonzero for zero bias, displays a nonmonotonic bias dependence, changing sign between $V_{\text {on }}^{\uparrow}$ and $V_{\text {on }}^{\downarrow}$, similar to that of the exchange field in quantum dots connected to FM leads. ${ }^{22}$ It is important to note that both $T_{i, \|}$ and $T_{i, \perp}$ are strongly enhanced for $N_{c}=7$ ASs in Fig. 6(d), even though the corresponding spinpolarized currents are smaller than those for $N_{c}=4$ ASs. Thus, the enhancement of the local spin torque is not associated with a corresponding enhancement of the spinpolarized currents. This result clearly demonstrates that the underlying mechanism that controls the local spin-transfer torque enhancement is the close proximity of the majority and minority QWS energies of different quantum number, $E^{n^{\uparrow}} \approx E^{n^{\prime} \downarrow}$, within the bias energy window. This in turn enhances the spin mixing $\sigma \leftrightarrow \bar{\sigma}$ in the central FM, when electrons tunnel resonantly through the SPQWSs. The enhancement of the local spin-transfer torque is independent of the parity of the QWS wave functions. This spin-transfer torque enhancement may have technological applications since the CIMS may be facilitated under such conditions.

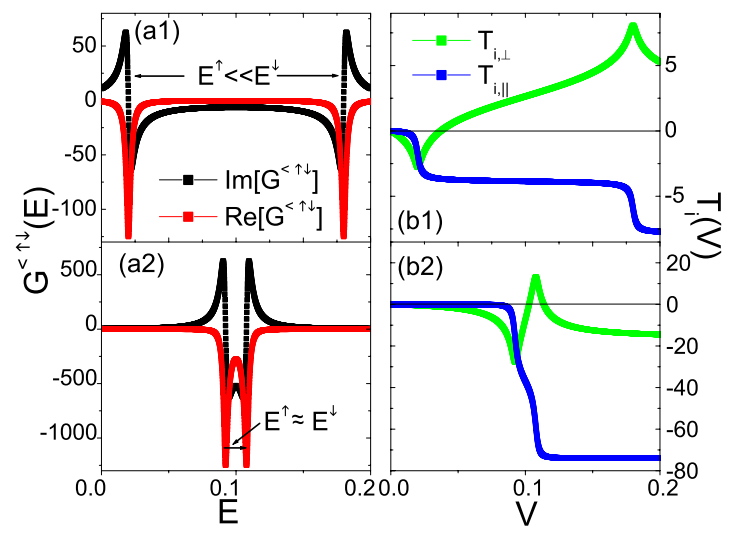

FIG. 7. (Color online) [(a1) and (a2)] Energy dependence of the real (red squares) and imaginary (black squares) parts of $G^{<\uparrow \downarrow}$ for $\left(E^{\uparrow} \ll E^{\downarrow}\right)$ and $\left(E^{\uparrow} \approx E^{\downarrow}\right)$, respectively; [(b1) and (b2)] bias dependence of $T_{i, \|}$ (blue squares) and of $T_{i, \perp}$ (green squares) corresponding to (a1) and (a2), respectively.

In order to elucidate the role of the SPQWSs on the enhancement and bias dependence of the local spin torque, we have used a simple model of a central FM region consisting of a single site coupled weakly to the leads. We have shown that, to leading order in the coupling parameter $t_{C}$, the on-site spin off-diagonal nonequilibrium Green's function is

$$
G^{<\uparrow \downarrow} \approx i \sum_{\alpha=L, R} f_{\alpha} G_{r}^{\uparrow \uparrow} \Sigma_{\alpha}^{\uparrow \downarrow} G_{a}^{\downarrow \downarrow} .
$$

Here, $\Sigma_{\alpha}^{\uparrow \downarrow}=t_{C}^{2} \pi\left[N_{\alpha}^{\uparrow}-N_{\alpha}^{\downarrow}\right] \sin (\theta)$ is the self-energy due to the leads, and $N_{\alpha}^{\uparrow(\downarrow)}$ is the surface density of states of the $L(R)$ leads, which, for simplicity, are taken to be energy independent. In the weak coupling regime, the retarded (advanced) Green's functions of the coupled system, $G_{r(a)}^{\sigma \sigma}$, can be approximated with those of the uncoupled system, $g_{r(a)}^{\sigma \sigma}$, i.e., $G_{r(a)}^{\sigma \sigma} \approx g_{r(a)}^{\sigma \sigma}=\left[E-E^{\sigma} \pm i \eta\right]^{-1}$, where $\eta$ is taken to be spin independent. Therefore, the spin-transfer torque components, determined by the nonequilibrium on-site magnetization, ${ }^{21}$ are

$$
T_{i, \|} \propto \int_{-\infty}^{e V} \frac{\eta\left(E^{\uparrow}-E^{\downarrow}\right)}{\left(\left(E-E^{\uparrow}\right)^{2}+\eta^{2}\right)\left(\left(E-E^{\downarrow}\right)^{2}+\eta^{2}\right)} d E
$$

and

$$
T_{i, \perp} \propto \int_{-\infty}^{e V} \frac{\left(E-E^{\uparrow}\right)\left(E-E^{\downarrow}\right)+\eta^{2}}{\left(\left(E-E^{\uparrow}\right)^{2}+\eta^{2}\right)\left(\left(E-E^{\downarrow}\right)^{2}+\eta^{2}\right)} d E .
$$

The energy dependence of the real (red squares) and imaginary (black squares) parts of $G^{<\uparrow \downarrow}$ for $\left(E^{\uparrow} \ll E^{\downarrow}\right)$ and $\left(E^{\uparrow} \approx E^{\downarrow}\right)$ are shown in panels (a1) and (a2) of Fig. 7, respectively. The corresponding bias dependence of $T_{i, \|}$ (blue squares) and of $T_{i, \perp}$ (green squares) are shown in panels (b1) and (b2) of Fig. 7, respectively. One can clearly see that the overall bias dependence of this simple model reproduces qualitatively that displayed in Figs. 6(c) and 6(d). More specifically, the switch-on behavior for $T_{i, \|}$ and the sign change of $T_{i, \perp}$ with bias are associated with the relative position of 


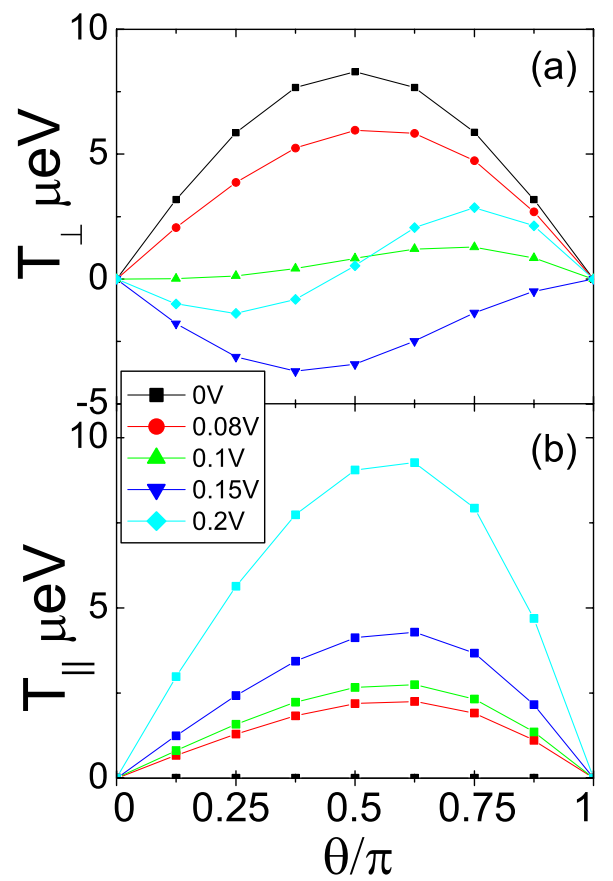

FIG. 8. (Color online) (a) Angular dependence of the net (a) fieldlike torque and (b) spin-transfer torque for $N_{c}=4$ and various values of bias.

the majority and minority QWS energies which lie within the bias energy window. When $E^{\uparrow} \approx E^{\downarrow}$, both components of the spin-transfer torque are dramatically enhanced, as shown in Fig. 7(a2), due to the presence of higher-order poles in Eqs. (12) and (13).

The net spin torque components, $T_{\|(\perp)}=\Sigma_{i \varepsilon C} T_{i, \|(\perp)}$, are not as strongly enhanced as the local torques, $T_{i, \|}$ and $T_{i, \perp}$, which oscillate as a function of site $i$ due to the electron precession in the central FM (Fig. 5). The angular dependence of the net fieldlike torque $T_{\perp}$ and the spin-transfer torque $T_{\|}$is shown in Figs. 8(a) and 8(b), respectively, for $N_{c}=4$ ASs and for various bias values. For zero bias, $T_{\perp}$ displays sinusoidal behavior, which, however, can change dramatically upon increasing the bias. The net fieldlike torque can be expressed $^{23,24}$ as

$$
T_{\perp}=-\partial E_{\mathrm{XC}}(\theta) / \partial \theta
$$

where

$$
E_{\mathrm{XC}}(\theta)=-J_{1} \cos (\theta)-J_{2} \cos ^{2}(\theta)+\cdots
$$

is the effective exchange coupling energy ${ }^{25}$ between $\mathbf{M}_{C}$ and $\mathbf{M}_{L(R)}$. The out of equilibrium interlayer exchange coupling has terms related to the spin-polarized tunnel current that can dominate and alter the coupling behavior under certain bias conditions. ${ }^{26}$ Here, $J_{1}$ and $J_{2}$ are the nonequilibrium bilinear and biquadratic effective exchange couplings, respectively,

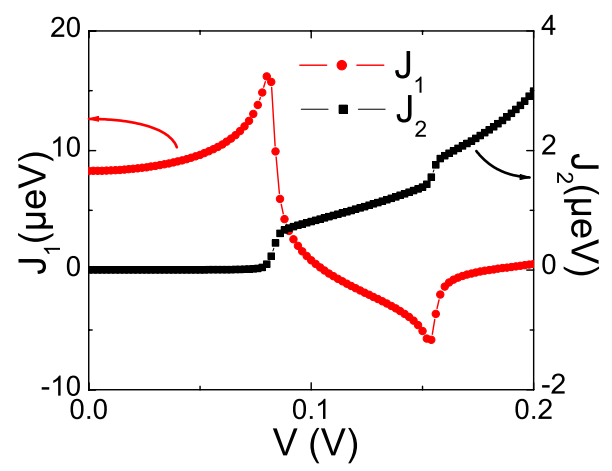

FIG. 9. (Color online) Bias dependence of the nonequilibrium effective bilinear, $J_{1}$, and biquadratic, $J_{2}$, interlayer exchange couplings for $N_{c}=4$.

which are determined by fitting the angular dependence of $T_{\perp}(\theta)$ in Fig. 8 to the above expression for various biases. In Fig. 9, we show the bias dependence of $J_{1}$ and $J_{2}$ for $N_{c}=4$ ASs. It is important to note that $J_{1}$ (red circles) reverses its sign with bias, similar to $T_{i, \perp}(V)$ in Fig. 6(c). On the other hand, the bias dependence of $J_{2}$ (black squares) exhibits the switch-on bias behavior found for $T_{i, \|}(V)$. Hence, there is a range of bias where $J_{2}>J_{1}$, favoring perpendicular alignment of $\mathbf{M}_{C}$ and $\mathbf{M}_{L(R)} \cdot{ }^{25}$ In contrast, the angular dependence of the net $T_{\|}$exhibits a skewed sinusoidal behavior [Fig. $8(b)]$ for any bias, similar to that found in spin valves.

\section{CONCLUSION}

In summary, we demonstrate that the local spin-transfer torque can be dramatically enhanced when the majority and minority QWSs of different quantum numbers are in close energetic proximity and lie within the bias energy window. This enhancement may in turn lead to reduction of the critical current necessary for CIMS in magnetic memories. The spin-torque enhancement criterion may be achieved by controlling the SPQWSs through an external magnetic field or spin-dependent barriers. The SPQWSs tune selectively the bias dependence of the spin-transfer and fieldlike components of the local and the net spin torque. This results to an anomalous angular behavior of $T_{\perp}$ due to the bias interplay of the bilinear and biquadratic effective exchange couplings. Future work will be aimed to include the results for the local spin torques of these calculations as an input into the Landau-Lifshitz-Gilbert equation to calculate the critical current for the CIMS.

\section{ACKNOWLEDGMENTS}

The research was supported by NSF-PREM Grant No. DMR-0611562, U.S. Army Grant No. W911NF-04-1-0058, and NSF-KITP Grant No. PHY99-07949. 
*ytheod@mail.ntua.gr

${ }^{1}$ I. Zŭtic, J. Fabian, and S. Das Sarma, Rev. Mod. Phys. 76, 323 (2004).

${ }^{2}$ J. C. Slonczewski, Phys. Rev. B 39, 6995 (1989).

${ }^{3}$ J. C. Slonczewski, J. Magn. Magn. Mater. 159, L1 (1996).

${ }^{4}$ L. Berger, Phys. Rev. B 54, 9353 (1996).

${ }^{5}$ S. Zhang, P. M. Levy, and A. Fert, Phys. Rev. Lett. 88, 236601 (2002).

${ }^{6}$ M. D. Stiles and J. Miltat, Spin Dynamics in Confined Magnetic Structures III (Springer, Berlin, 2006), p. 225.

${ }^{7}$ A. A. Tulapurkar, Y. Suzuki, A. Fukushima, H. Kubota, H. Maehara, K. Tsunekawa, D. D. Djayaprawira, N. Watanabe, and S. Yuasa, Nature (London) 438, 339 (2005).

${ }^{8}$ J. C. Sankey, P. M. Braganca, A. G. F. Garcia, I. N. Krivorotov, R. A. Buhrman, and D. C. Ralph, Phys. Rev. Lett. 96, 227601 (2006).

${ }^{9}$ G. D. Fuchs, J. A. Katine, S. I. Kiselev, D. Mauri, K. S. Wooley, D. C. Ralph, and R. A. Buhrman, Phys. Rev. Lett. 96, 186603 (2006).

${ }^{10}$ S. Urazhdin, H. Kurt, M. AlHajDarwish, Norman O. Birge, W. P. Pratt, Jr., and J. Bass, J. Appl. Phys. 97, $10 \mathrm{C701}$ (2005).

${ }^{11}$ Y. Huai, F. J. Albert, P. Nguyen, M. Pakala, and T. Valet, Appl. Phys. Lett. 84, 3118 (2004).

${ }^{12}$ M. Hosomi, H. Yamagishi, T. Yamamoto, K. Bessho, Y. Higo, K. Yamane, H. Yamada, M. Shoji, H. Hachino, C. Fukumoto, H. Nagao, and H. Kano, Tech. Dig. - Int. Electron Devices Meet. 2005, 459 (2005).

${ }^{13}$ A. G. Petukhov, A. N. Chantis, and D. O. Demchenko, Phys. Rev.
Lett. 89, 107205 (2002).

${ }^{14}$ Z.-Y. Lu, X.-G. Zhang, and S. T. Pantelides, Phys. Rev. Lett. 94, $207210(2005)$

${ }^{15}$ Z.-M. Zeng, X.-F. Han, W.-S. Zhan, Y. Wang, Z. Zhang, and S. Zhang, Phys. Rev. B 72, 054419 (2005).

${ }^{16}$ T. Nozaki, N. Tezuka, and K. Inomata, Phys. Rev. Lett. 96, 027208 (2006).

${ }^{17}$ K. Yakushiji, F. Ernult, H. Imamura, K. Yamane, S. Mitani, K. Takanashi, S. Takahashi, S. Maekawa, and H. Fujimori, Nat. Mater. 4, 57 (2005).

${ }^{18}$ J. Hong, R. Q. Wu, and R. B. Muniz, J. Magn. Magn. Mater. 270, 298 (2004)

${ }^{19}$ S. Datta, Electronic Transport in Mesoscopic Systems (Cambridge University Press, Cambridge, 1995).

${ }^{20}$ I. Theodonis, Nicholas Kioussis, Alan Kalitsov, Mairbek Chshiev, and W. H. Butler, Phys. Rev. Lett. 97, 237205 (2006).

${ }^{21}$ A. Kalitsov, I. Theodonis, N. Kioussis, M. Chshiev, W. H. Butler, and A. Vedyayev, J. Appl. Phys. 99, 08G501 (2006).

${ }^{22}$ M. Braun, J. Kŏnig, and J. Martinek, Phys. Rev. B 70, 195345 (2004).

${ }^{23}$ S. O. Demokritov, J. Phys. D 31, 925 (1998).

${ }^{24}$ J. d'Albuquerque e Castro, M. S. Ferreira, and R. B. Muniz, Phys. Rev. B 49, 16062 (1994).

${ }^{25}$ J. C. Slonczewski, J. Appl. Phys. 73, 5957 (1993); J. Magn. Magn. Mater. 150, 13 (1995).

${ }^{26}$ C. Heide, R. J. Elliott, and N. S. Wingreen, Phys. Rev. B 59, 4287 (1999); C.-Y. You and S. D. Bader, J. Magn. Magn. Mater. 195, 488 (1999). 